\title{
Dosimetric comparison between proton beam therapy and photon radiation therapy for locally advanced esophageal squamous cell carcinoma
}

Yasuhiro Hirano', Masakatsu Onozawa', Hidehiro Hojo ${ }^{1}$, Atsushi Motegi', Sadatomo Zenda', Kenji Hotta', Shunsuke Moriya', Hidenobu Tachibana', Naoki Nakamura', Takashi Kojima² and Tetsuo Akimoto ${ }^{1 *}$

\begin{abstract}
Background: The purpose of this study was to perform a dosimetric comparison between proton beam therapy (PBT) and photon radiation therapy in patients with locally advanced esophageal squamous cell carcinoma (ESCC) who were treated with PBT in our institution. In addition, we evaluated the correlation between toxicities and dosimetric parameters, especially the doses to normal lung or heart tissue, to clarify the clinical advantage of PBT over photon radiation therapy.

Methods: A total of 37 consecutive patients with Stage III thoracic ESCC who had received PBT with or without concurrent chemotherapy between October 2012 and December 2015 were evaluated in this study. The dose distributions of PBT were compared with those of dummy 3-dimensional conformal radiation therapy (3DCRT) and Intensity Modulated Radiation Therapy (IMRT), focusing especially on the doses to organs at risk, such as normal lung and heart tissue.

Results: Of the 37 patients, the data from 27 patients were analyzed. Among these 27 patients, four patients (15\%) developed grade 2 pericardial effusion as a late toxicity. None of the patients developed grade 3 or worse acute or late pulmonary and cardiac toxicities. When the dosimetric parameters between PBT and planned 3DCRT were compared, all the PBT domestic variables for the lung dose except for lung V10 GyE and V15 GyE were significantly lower than those for the dummy 3DCRT plans, and the PBT domestic variables for the heart dose were also significantly lower than those for the dummy 3DCRT plans. When the PBT and IMRT plans were compared, all the PBT domestic variables for the doses to the lung and heart were significantly lower than those for the dummy IMRT plans. Regarding the correlation between the grades of toxicities and the dosimetric parameters, no significant correlation was seen between the occurrence of grade 2 pericardial effusion and the dose to the heart.

Conclusions: When the dosimetric parameters of the dose distributions for the treatment of patients with locally advanced stage III ESCC were compared between PBT and 3DCRT or IMRT, PBT enabled a significant reduction in the dose to the lung and heart, compared with 3DCRT or IMRT.
\end{abstract}

Keywords: Proton beam therapy, IMRT, 3DCRT, Locally advanced esophageal cancer, In silico dose distribution

\footnotetext{
* Correspondence: takimoto@east.ncc.go.jp

'Division of Radiation Oncology and Particle Therapy, National Cancer Center

Hospital East, 6-5-1, Kashiwanoha, Kashiwa, Chiba 277-8577, Japan

Full list of author information is available at the end of the article
} 


\section{Background}

One standard treatment option for stage II-III esophageal squamous cell carcinoma (ESCC) is neoadjuvant chemotherapy followed by surgical resection, and the 5 -year overall survival rate is reported to be $36.8 \%-61 \%$ [1-3]. Chemoradiotherapy (CRT) is also a curative-intent non-surgical treatment option for resectable ESCC, especially for patients who refuse surgical resection or are unsuited for surgical resection. The results of the Japan Clinical Oncology Group (JCOG) 9906, a Phase II study that evaluated CRT for the patients with stage II-III ESCC, showed an initial complete response rate of $62.2 \%$ and a 5-year survival rate of $36.8 \%$ [4]. However, 4 (5.3\%) related deaths occurred because of late toxicities including pneumonitis $(n=2)$, pericarditis $(n=1)$, and pleural effusion $(n=1)$. Long-term cardiopulmonary toxicities sometimes caused life-threatening events or death in patients who have received CRT, and the main causes for the development of cardiopulmonary toxicities are excessive radiation doses to normal lung and heart tissue, in addition to the combination of chemotherapy with radiation therapy. Therefore, it is important to reduce the incidence and severity of late toxicities, since this would lead to an improved quality of life (QOL) for patients who are able to achieve disease control.

Particle therapy including proton beam therapy (PBT) has a unique physical characteristic, called the Bragg peak, and can deliver a high dose to the tumor while sparing the surrounding normal tissues. An in silico dose distribution comparison between photon radiation therapy and PBT for esophageal cancer shows that proton beam therapy has clear therapeutic advantages, especially a dose reduction to at-risk organs, over conventional external radiotherapy [5]. Based on these backgrounds, we have been applying PBT with concurrent chemotherapy for the treatment of patients with ESCC since 2012. The purpose of the present study was to perform a dosimetric comparison between PBT and photon radiation therapy in patients who were treated with PBT for locally advanced esophageal ESCC in our institution. We also evaluated the correlation between the grade and/or incidence of toxicities and dosimetric parameters, especially the doses to normal lung or heart tissue, to clarify the clinical advantages of $\mathrm{PBT}$ over photon radiation therapy.

\section{Methods}

\section{Patients}

Approval for this study was obtained from the National Cancer Center Institutional Review Board. Consecutive patients with thoracic ESCC who received PBT with or without concurrent chemotherapy between October 2012 and December 2015 were enrolled as candidates for this study. Among these, we selected 37 consecutive patients with Stage III thoracic ESCC for dosimetric comparison between PBT and photon radiation therapy because the rationale for the coverage of the radiation field, including the elective nodal region, differs according to disease stage, and this might have resulted in differences in the doses to risk organs, such as the lung and heart.

\section{Pretreatment evaluation}

Clinical staging was based on the American Joint Committee on Cancer staging, 7th edition. The staging evaluations included a barium swallow test, an endoscopy examination of the esophagus and stomach, and a computed tomography examination of the neck, chest, and abdomen. When necessary, 18F-fluorodeoxyglucose positron emission tomography was also performed.

\section{Treatment details}

The chemotherapy regimen consisted of 2 cycles of cisplatin (CDDP) $\left(70 \mathrm{mg} / \mathrm{m}^{2}\right)$ on day 1 and the continuous infusion of fluorouracil (FU) $\left(700 \mathrm{mg} / \mathrm{m}^{2}\right)$ on days 1 to 4 repeated every 4 weeks, 2 cycles of CDDP $\left(75 \mathrm{mg} / \mathrm{m}^{2}\right)$ on day 1 and the continuous infusion of FU $\left(1000 \mathrm{mg} / \mathrm{m}^{2}\right)$ on days 1 to 4 repeated every 4 weeks, 2 cycles of nedaplatin $\left(80-90 \mathrm{mg} / \mathrm{m}^{2}\right)$ on day 1 and the continuous infusion of $\mathrm{FU}$ $\left(800 \mathrm{mg} / \mathrm{m}^{2}\right)$ on days 1 to 5 repeated every 4 weeks, or FU $\left(700 \mathrm{mg} / \mathrm{m}^{2}\right)$ on days 1 to 4 repeated every 4 weeks, with concurrent PBT. The fractionated schema for PBT was $60 \mathrm{GyE}$ in 30 fractions over 6 weeks. Dose reduction, the postponement of either drug or the discontinuation of PBT was implemented to prevent a worsening of adverse events.

For the PBT simulation, all the patients were placed in a supine position. Simulation CT images were obtained in 3-mm thick slices. An in-house treatment planning system with a calculation grid size of $1.876 \mathrm{~mm}$ was used for the PBT treatment planning. The dose calculations were performed using the pencil beam dose calculation algorithm. The gross tumor volume (GTV) was defined as the primary tumor and clinically positive lymph nodes. The clinical target volume (CTV) encompassed the GTV and the subclinical tumor extension. For primary lesion, $2 \mathrm{~cm}$ expansion cranio-caudally and $3 \mathrm{~mm}$ expansion radially, and no CTV margin was added for nodal disease. The planning target volume (PTV) covered the CTV with a 5-mm in all directions. A total dose of $60 \mathrm{GyE}$ in 30 fractions was given to the primary tumor and the metastatic lymph nodes. The dose constraints for the organs at risk (OARs) were as follows: normal lung V20 GyE (percentage of the normal lung volume irradiated with more than $20 \mathrm{GyE})<20 \%$, heart V30 GyE (percentage of the heart volume irradiated with more than $30 \mathrm{GyE})<46 \%$ and mean dose $<26$ GyE, and a spinal cord Dmax (maximum point dose) of 48 GyE. The beam output was modulated with a relative biologic effectiveness (RBE) of 1.1, based on a previous animal examination [6]. 


\section{Dosimetric analysis}

The dosimetric data for the OARs were collected from each treatment plan. For normal lung tissue, the V20, 15,10 , and $5 \mathrm{GyE}$ and the mean dose were calculated. For the heart, the V40, 30, 20, and $10 \mathrm{GyE}$ and the mean dose were calculated. For the spinal cord, the maximum point dose was calculated. Conformity index (CI) determined as the volume of the $90 \%$ prescription isodose surface divided by PTV was also evaluated. To compare the dosimetric parameters of the dose distribution between PBT and 3-dimensional conformal radiation therapy (3DCRT) and between PBT and intensitymodulated radiation therapy (IMRT), dummy 3DCRT plans and IMRT plans were created using the same simulated computed tomography (CT) images, structures and dose prescription settings for PBT. 3DCRT, IMRT and PBT plans were normalized to cover $50 \%$ of the PTV with $100 \%$ of the prescription dose.

In the dummy IMRT planning, the plan was approved when $50 \%$ of the PTV received $100 \%$ of the prescribed dose (D50 $=100 \%$ dose prescription), after eliminating hot spots receiving $\geq 110 \%$ of the prescribed dose. Dose constraints for the normal lung (V20 Gy), the heart (mean dose), and the spinal cord (maximum dose) were set at $<30 \%,<50 \mathrm{~Gy}$, and $<45 \mathrm{~Gy}$, respectively. All the plans were generated using 5-treatment beams with 6-MV X-rays. IMRT delivery was performed using the sliding window technique with the Varian Millennium 120-MLC. AAA was used as the dose calculation engine.

The dummy 3DCRT and IMRT plans were created using the Xio version 5.0 (Elekta, Sweden) and the Eclipse version 11.0 treatment planning system (Varian Medical System, USA), respectively. In the dummy 3DCRT plans, photon energies of $6-\mathrm{MV}$ and $10-\mathrm{MV}$ were used. For the dose calculation of the 3DCRT plan, the superposition method with inhomogeneous correction was used, with a grid size of $2 \mathrm{~mm}$. The plans were approved when the CTV could receive at least 95\% without exceeding the dose constraints for the OARs, as described previously.

\section{Toxicity assessment}

Adverse events caused by PBT with or without concurrent chemotherapy were evaluated based on the Common Terminology Criteria for Adverse Events (CTCAE), version 4.0. In this study, non-hematological toxicities including pulmonary and cardiac adverse events were mainly evaluated. Acute adverse events were assessed within 2 months from the first day of PBT and were graded based on the worst symptoms experienced during this period. Symptoms or events caused by PBT and that occurred at least 3 months after the first day of PBT were evaluated as late adverse events.
Table 1 Patient characteristics

\begin{tabular}{|c|c|c|}
\hline Characteristic & Patients $(n=27)$ & $(\%)$ \\
\hline$\overline{M a l e}$ & 23 & 85.2 \\
\hline Female & 4 & 14.8 \\
\hline \multicolumn{3}{|l|}{ Age (y) } \\
\hline Range & $50-90$ & \\
\hline Median & 70 & \\
\hline \multicolumn{3}{|l|}{ T stage } \\
\hline T3 & 25 & 92.6 \\
\hline T4 & 2 & 7.4 \\
\hline \multicolumn{3}{|l|}{ N stage } \\
\hline NO & 0 & 0 \\
\hline $\mathrm{N} 1$ & 16 & 59.3 \\
\hline N2 & 10 & 37.0 \\
\hline N3 & 1 & 3.7 \\
\hline \multicolumn{3}{|l|}{ Stage } \\
\hline$\| I A$ & 15 & 55.6 \\
\hline$\| \mid \mathrm{B}$ & 9 & 33.3 \\
\hline$\| I I C$ & 3 & 11.1 \\
\hline \multicolumn{3}{|l|}{ Location of the lesion } \\
\hline Ut & 5 & 18.5 \\
\hline Mt & 9 & 33.3 \\
\hline Mt./Lt & 6 & 22.2 \\
\hline $\mathrm{Lt}$ & 5 & 18.5 \\
\hline $\mathrm{Lt} / \mathrm{Ae}$ & 2 & 7.4 \\
\hline \multicolumn{3}{|l|}{ Chemotherapy } \\
\hline $5-\mathrm{Fu}+\mathrm{CDDP}$ & 16 & 59.3 \\
\hline 5-Fu + nedaplatin & 7 & 25.9 \\
\hline $5-\mathrm{Fu}$ & 1 & 3.7 \\
\hline Radiotherapy alone & 3 & 11.1 \\
\hline
\end{tabular}

Abbreviations: Ut upper thoracic esophagus, Mt. middle thoracic esophagus, $L t$ lower thoracic esophagus, Ae abdominal esophagus, Fu fluorouracil, CDDP cisplatin

\section{Statistical analysis}

The survival analysis was performed by the Kaplan-Meier method. Overall survival was calculated from the first day of PBT to death due to any cause. Progressionfree survival was defined as the time from the first day of

Table 2 Acute and late pulmonary and cardiac adverse events

\begin{tabular}{lllll}
\hline & Grade1 & Grade2 & Grade3 & Grade4-5 \\
\hline Acute toxicity & $7(26 \%)$ & 0 & 0 & 0 \\
Pneumonitis & - & $1(4 \%)$ & 0 & 0 \\
Pericardial effusion & - & & \\
Late toxicity & $17(63 \%)$ & 0 & 0 & 0 \\
Pneumonitis & $5(19 \%)$ & 0 & 0 & 0 \\
Pleural effusion & - & $4(15 \%)$ & 0 & 0 \\
Pericardial effusion & & & & \\
\hline
\end{tabular}




\section{a $\mathrm{PBT}$}
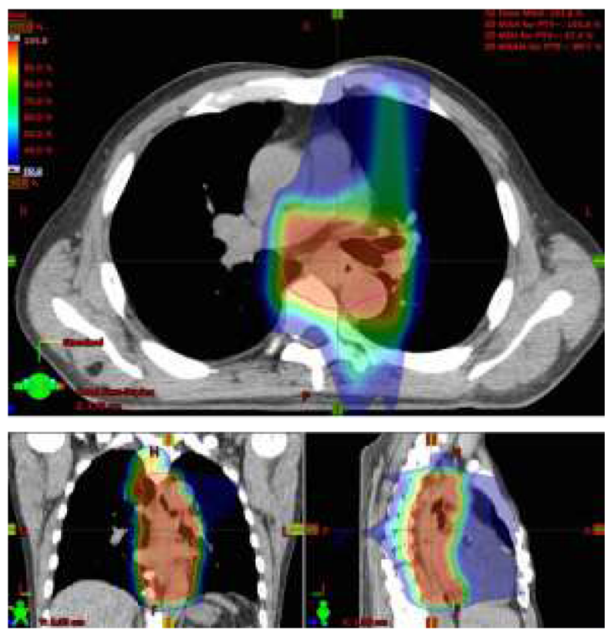

b 3DCRT (dummy)
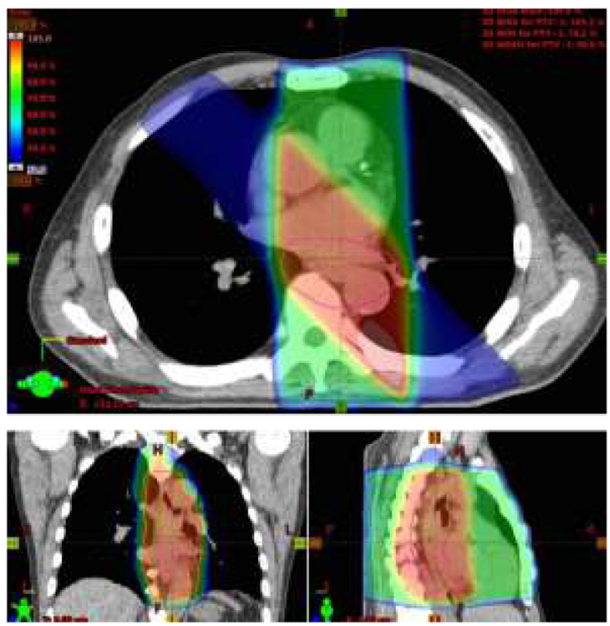

\section{IMRT (dummy)}
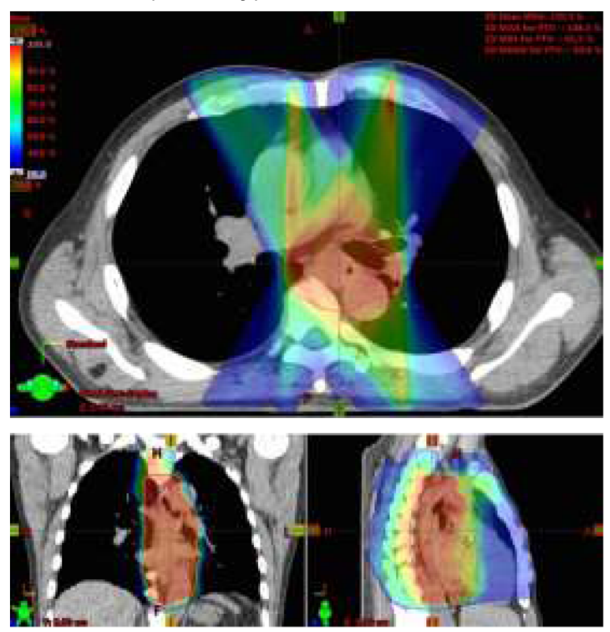

Fig. 1 Axial, sagittal, and coronal views of the dose distributions for T3 N1 middle thoracic esophageal cancer. a PBT, b dummy plan for 3DCRT using 4 portals, $\mathbf{c}$ dummy plan for IMRT. The red line represents the PTV. PBT, proton beam therapy; 3DCRT, 3-dimensional conformal radiation therapy; IMRT, intensity-modulated radiation therapy; PTV, planning target volume

PBT to that of disease progression, evidence of residual disease or death from any cause.

The statistical analysis was performed using IBM SPSS Statistics 22. A paired $t$-test was performed to compare the doses to the OARs.

\section{Results}

\section{Patient characteristics}

Of the 37 patients, data from 27 patients were analyzed retrospectively. Ten of the 37 patients were excluded from the study because 3 patients received a total dose of $66 \mathrm{GyE}$ in 33 fractions, two patients received a total dose of 50.4 GyE in 28 fractions, and five patients received a combination of x-rays and PBT. The characteristics of the 27 patients who were included in the analysis are summarized in Table 1. Of these 27 patients, three patients received PBT alone because two patients refused to receive chemotherapy and one patient was not a candidate for chemotherapy because of a poor physical condition. These patients didn't receive any chemotherapy including neoadjuvant or adjuvant setting.

\section{Survival}

The median follow-up period was 442 days (range, 69-1, 468 days) for all the eligible patients. The median follow-up period for the censored cases was 445 days (range, 69-1, 468 days). The overall survival and progression-free survival at 1 year after completion of PBT were 90.8 and $40.6 \%$, respectively.

\section{Toxicities}

Acute and late pulmonary and cardiac adverse events are shown in Table 2. One case required an interruption in PBT because of grade 3 esophagitis however, none of the patients required PBT interruption because of pulmonary or cardiac adverse events. Four patients (15\%) developed grade 2 pericardial effusion as a late toxicity. The median period from the last day of PBT until the development of grade 2 pericardial effusion was 179 days, ranging from 56 to 280 days. At the time of analysis, none of the patients had developed grade 3 or worse, acute or late, pulmonary or cardiac toxicities.

\section{Dosimetric analysis}

Dummy plans for 3DCRT at the same radical dose as PBT were generated for nine patients using foue portal 
Table 3 Dosimetric comparison between PBT and 3DCRT, PBT and IMRT

\begin{tabular}{|c|c|c|c|c|c|c|c|}
\hline End point & DVH parameters & Technique & Mean & SD & SE mean & $\begin{array}{l}p \text { value. } \\
\text { (vs. 3DCRT) }\end{array}$ & $\begin{array}{l}p \text { value. } \\
\text { (vs. IMRT) }\end{array}$ \\
\hline \multirow[t]{15}{*}{ Lung } & \multirow[t]{3}{*}{ V20 (\%) } & PBT & 11.6607 & 5.18604 & .99805 & \multirow[t]{3}{*}{.001} & \multirow[t]{3}{*}{$<0.01$} \\
\hline & & 3DCRT & 16.9652 & 5.64638 & 1.08665 & & \\
\hline & & IMRT & 17.7941 & 5.19620 & 1.00001 & & \\
\hline & \multirow[t]{3}{*}{ V15 (\%) } & PBT & 17.8696 & 7.22321 & 1.39011 & \multirow[t]{3}{*}{.052} & \multirow[t]{3}{*}{.006} \\
\hline & & 3DCRT & 21.6615 & 6.75645 & 1.30028 & & \\
\hline & & IMRT & 22.9630 & 5.64911 & 1.08717 & & \\
\hline & \multirow[t]{3}{*}{ V10 (\%) } & PBT & 21.3307 & 9.07254 & 1.74601 & \multirow[t]{3}{*}{.058} & \multirow[t]{3}{*}{$<0.01$} \\
\hline & & 3DCRT & 25.7222 & 7.46624 & 1.43688 & & \\
\hline & & IMRT & 30.0181 & 6.84990 & 1.31826 & & \\
\hline & \multirow[t]{3}{*}{ V5 (\%) } & PBT & 25.2478 & 10.77897 & 2.07441 & \multirow[t]{3}{*}{.005} & \multirow[t]{3}{*}{$<0.01$} \\
\hline & & 3DCRT & 33.0893 & 8.79872 & 1.69331 & & \\
\hline & & IMRT & 45.1811 & 10.52645 & 2.02582 & & \\
\hline & \multirow[t]{3}{*}{ Mean dose (Gy) } & PBT & 5.8322 & 2.24148 & .43137 & \multirow[t]{3}{*}{$<0.01$} & \multirow[t]{3}{*}{$<0.01$} \\
\hline & & 3DCRT & 8.2111 & 2.14654 & .41310 & & \\
\hline & & IMRT & 9.4778 & 2.17052 & .41772 & & \\
\hline \multirow[t]{12}{*}{ Heart } & \multirow[t]{3}{*}{ V40 (\%) } & PBT & 16.3059 & 11.42262 & 2.19828 & \multirow[t]{3}{*}{$<0.01$} & \multirow[t]{3}{*}{0.012} \\
\hline & & 3DCRT & 48.1837 & 21.03337 & 4.04787 & & \\
\hline & & IMRT & 26.5770 & 16.91562 & 3.25541 & & \\
\hline & \multirow[t]{3}{*}{ V30 (\%) } & PBT & 22.0252 & 14.45506 & 2.78188 & \multirow[t]{3}{*}{$<0.01$} & \multirow[t]{3}{*}{$<0.01$} \\
\hline & & 3DCRT & 56.1574 & 21.49217 & 4.13617 & & \\
\hline & & IMRT & 50.7126 & 23.40755 & 4.50479 & & \\
\hline & \multirow[t]{3}{*}{ V20 (\%) } & PBT & 38.4963 & 23.91227 & 4.60192 & \multirow[t]{3}{*}{$<0.01$} & \multirow[t]{3}{*}{$<0.01$} \\
\hline & & 3DCRT & 64.1178 & 23.03079 & 4.43228 & & \\
\hline & & IMRT & 69.3259 & 26.97548 & 5.19143 & & \\
\hline & \multirow[t]{3}{*}{ Mean dose (Gy) } & PBT & 17.6089 & 9.68207 & 1.86331 & \multirow[t]{3}{*}{$<0.01$} & .001 \\
\hline & & 3DCRT & 30.9844 & 11.45297 & 2.20412 & & \\
\hline & & IMRT & 9.4778 & 2.17052 & .41772 & & \\
\hline Spinal cord & Maximum dose (Gy) & PBT & 38.1400 & 4.96680 & .95586 & $<0.01$ & .155 \\
\hline & & 3DCRT & 47.2896 & 2.22998 & .42916 & & \\
\hline & & IMRT & 39.7119 & 2.64873 & .50975 & & \\
\hline $\mathrm{Cl}$ & & PBT & 1.8796 & .21319 & .04103 & $<0.01$ & $<0.01$ \\
\hline & & 3DCRT & 3.1886 & .61704 & .11875 & & \\
\hline & & IMRT & 1.6243 & 23493 & .04521 & & \\
\hline
\end{tabular}

SD standard deviation, $S E$ standard error, $\mathrm{Cl}$ conformity index

beams, while those of the other 18 patients were generated using more than four portal beams because of the difficulty in creating an acceptable dose to the spinal cord arising from the undesirable spatial relationship between the extent of the disease and the spinal cord. A representative PBT dose distribution, the dummy plan for 3DCRT, and the dummy plan for IMRT for a patient with T3 N1 middle thoracic esophageal cancer is shown in Fig. 1.

A paired $t$-test was performed to compare the dosimetric results between the PBT plans and the 3DCRT plans and between the PBT plans and the IMRT plans statistically (Table 3 ). When the dosimetric parameters between the PBT and 3DCRT plans were compared, all the PBT domestic variables regarding the lung dose except for the lung V10 GyE and V15 GyE were significantly lower than those of the dummy 3DCRT plans (Table 3). In addition, all the PBT domestic variables regarding the heart dose (mean dose, V20, V30, V40) were significantly lower than those of the dummy 3DCRT plans. When the dosimetric parameters were compared between the PBT and IMRT plans, all the PBT domestic variables regarding the dose to the 
lung and heart including the lung V10 and V15 GyE were significantly lower than those of the dummy IMRT plans (Table 3).

\section{Correlation between toxicities and dosimetric parameters} Regarding the correlation between the grades and/or incidence of toxicities and the dosimetric parameters, we evaluated the impact of the dose to the heart on the occurrence of grade 2 pericardial effusion, since four patients developed grade 2 pericardial effusion as a late toxicity and none of the patients developed grade 3 or severer late toxicities. However, no significant correlation between the occurrence of grade 2 pericardial effusion and the dose to heart, including the mean heart dose, heart V20, V30 and V40, was observed, although all the parameters in the patients who developed grade 2 pericardial effusion were slightly higher than those in the patients who did not (Table 4).

\section{Discussion}

When the dosimetric parameters between PBT and the 3DCRT or IMRT plans were compared, the present results demonstrated that PBT resulted in a significantly lower dose to the lung and heart, while providing an optimal dose to the treatment targets, compared with 3DCRT or IMRT for locally advanced stage III ESCC. In radiotherapeutic management for ESCC, concurrent CRT has been established as a standard treatment modality for patients with ESCC who are not suited to undergo surgical resection or who refuse surgery, and satisfactory clinical outcomes have been obtained [7-9]. However, late toxicities such as pleural effusion or pericardial effusion have been important issues that should be solved.

Table 5 summarizes the reported results of late toxicities after CRT in esophageal cancer [4, 10-14]. The main cardiopulmonary late toxicities were pleural effusion, pericardial effusion and radiation pneumonitis, and

Table 4 Correlation between late pericardial effusion toxicity and dose to heart

\begin{tabular}{llllll}
\hline $\begin{array}{l}\text { DVH } \\
\text { parameters }\end{array}$ & $\begin{array}{l}\text { Pericardial Effusion } \\
\text { (Grade) }\end{array}$ & Mean & SD & SE mean & $p$ value \\
\hline V40 (\%) & 2 & 16.2850 & 13.53387 & 6.76694 & .997 \\
& 0 & 16.3096 & 11.36759 & 2.37031 & \\
V30 (\%) & 2 & 23.2550 & 19.36524 & 9.68262 & .858 \\
& 0 & 21.8113 & 13.98136 & 2.91531 & \\
V20 (\%) & 2 & 42.2300 & 32.64928 & 16.32464 & .742 \\
& 0 & 37.8470 & 22.96571 & 4.78868 & \\
Mean dose & 2 & 19.1400 & 13.02880 & 6.51440 & .739 \\
(Gy) & & 17.3426 & 9.33480 & 1.94644 & \\
& 0 & & & &
\end{tabular}

the incidences of grade 3 or worse pericardial effusion and radiation pneumonitis were around $10 \%$ and $3 \%$, respectively. However, the occurrence of cardiopulmonary late toxicities impairs the QOL of patients, although the symptoms are usually improved by optimal medical interventions. In addition, several authors have described that cardiopulmonary complications sometimes become life-threatening $[11,13]$. Ishikura et al. reported that two patients died because of acute myocardial infarction among 78 patients who achieved complete remission, and the cause of death of 8 patients might have been related to cardiopulmonary toxicity [11]. Morota et al. also reported that one patient died of heart failure among 69 patients treated with CRT for esophageal cancer [13]. Considering these clinical outcomes, the occurrence of severe late toxicities should be reduced and avoided. (Here is the location of Table 5.)

As described above, the main reasons for the development of pulmonary and/or cardiac late toxicities is thought to be an excessive dose to OARs such as the heart and lung, and the intensification of treatment through combined concurrent chemotherapy also affects the occurrence of severe pulmonary and/or cardiac late toxicities. However, the intensity of CRT must be maintained to obtain satisfactory clinical outcomes, including the initial response and long-term outcomes, because combined concurrent chemotherapy has been established as a standard treatment for locally advanced ESCC. Thus, reducing the radiation dose to the lung and heart would be an important and effective approach to decreasing the severity or incidence of treatment-related toxicities caused by CRT.

The current standard beam arrangement of 3DCRT for esophageal cancer is 4 beams, especially with a heavier weighting in the AP/PA direction, and this increases the dose to the heart and spinal cord. To reduce the dose to OARs, a multi-portal technique has been tested, and an improvement in the incidence of severe late cardiopulmonary toxicities has been obtained. Kato et al. reported in a study of CRT for stage II-III ESCC using a multi-field technique that late cardiopulmonary toxicities included grade 3 pneumonitis (5.9\%), but none of the cases had cardiac adverse events of grade 3 or worse severity [10]. Thus, the application of the multi-field technique was useful for reducing the dose to the heart.

An alternative, effective approach to reducing the dose to OARs is the application of particle therapy, especially PBT, combined with chemotherapy. Charged particles such as PBT and heavy particles have Bragg peak properties, meaning that almost all of their energy can be deposited in the target volume with tissues beyond the tumor location receiving minimal doses [15]. Considering the anatomical relationship between the esophagus and the heart/lungs, the application of particle therapy is likely 


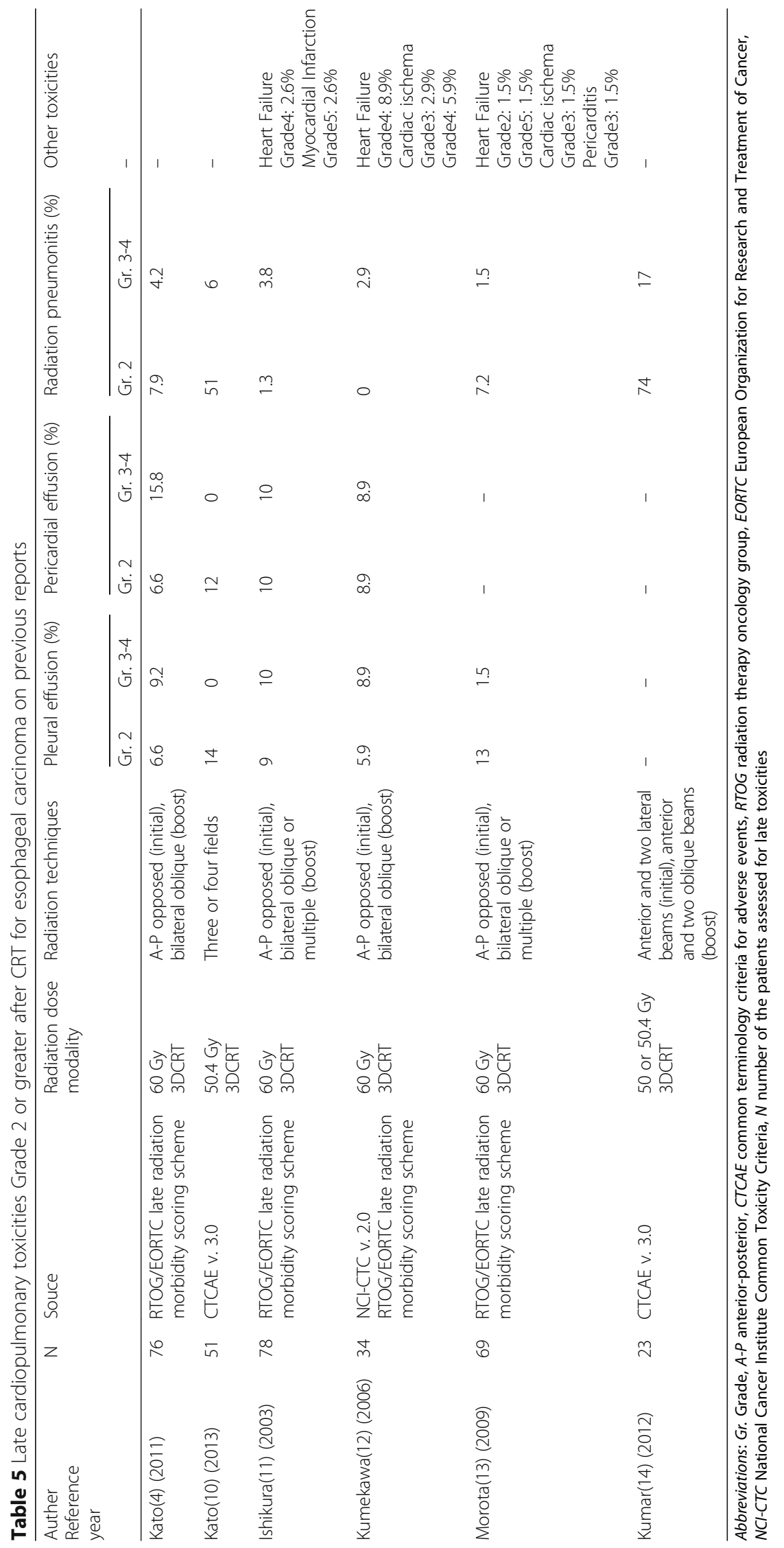


to reduce the dose to OARs, compared with photon radiation therapy. Actually, in a study comparing PBT with photon radiation therapy for esophageal cancer evaluated according to the tumor control probability (TCP) and the normal tissue complication probability (NTCP), the PBT plans were able to reduce the doses to structures of the lung and heart better like this study and appeared to have clear therapeutic advantages over photon radiation therapy [5]. Zhang et al. compared 4-dimensional computed tomography-based treatment plans with $\mathrm{PBT}$ or IMRT for distal esophageal cancer, and the application of PBT resulted in a more significant dose reduction to the lung than IMRT; however, no improvement in the dose to the heart was seen [16]. A limitation of the study by Zhang et al. was the small number of patients who were analyzed (15 patients); furthermore, the analysis was limited to patients with distal esophageal cancer, since the main histological type in the United States is adenocarcinoma, which usually develops in the distal esophagus. This difference in the locations of the primary tumors would affect the dose to OARs, such as the heart and lungs, because of the anatomical relationship between the esophagus and surrounding OARs. Therefore, the results of this study are expected to provide valuable information regarding the efficacy of PBT for patients with thoracic esophageal squamous cell carcinoma.

Regarding the incidence and severity of cardiopulmonary toxicities, the results of the current study showed a much lower incidence than those reported by other authors (Table 5). As described above, the main reason for this difference is likely the reduced dose to OARs that can be achieved using PBT. Actually, no significant correlation between the occurrence of grade 2 pericardial effusion and the dose to the heart, including the mean heart dose, was seen in this study, mainly because of the limited number of patients who developed grade 2 or severer toxicities. The further accumulation of data is warranted to clarify the correlation between the grade of toxicity and the dosimetric parameters of PBT.

The results of this study demonstrated the efficacy of PBT with concurrent chemotherapy for locally advanced ESCC based on a comparison of the dosimetric parameters to OARs between PBT and 3DCRT or IMRT plans. However, the present study had several limitations. First, the follow-up duration was not sufficient to evaluate long-term late toxicities. Second, the number of patients enrolled in this study was too small to evaluate the impact of the location of primary tumors on the dose to OARs. However, a strength of this study is that the analyzed patients were limited to consecutive patients with Stage III thoracic esophageal cancer, whose treatment strategies in terms of radiation field coverage were relatively uniform. Therefore, we believe that this study suggests that PBT with concurrent chemotherapy might be an effective treatment option for locally advanced esophageal squamous cell cancer. Based on the results of the dosimetric analysis, we are now conducting a phase I dose-escalating study of PBT with concurrent full-dose chemotherapy for patients with stage IB-III esophageal squamous cell carcinoma. In the future, large-scale prospectively randomized studies should be conducted to clarify the effectiveness of PBT with concurrent chemotherapy for the treatment of locally advanced esophageal cancer.

\section{Conclusions}

The results of this study demonstrated that PBT enabled a significant reduction in the dose to the lung and heart, compared with treatment plans for 3DCRT or IMRT, for locally advanced stage III ESCC when the dosimetric parameters between PBT and 3DCRT or IMRT plans were compared.

\section{Abbreviations}

3DCRT: 3-dimensional conformal radiation therapy; CDDP: Cisplatin; CRT: Chemoradiotherapy; CTV: Clinical target volume; ESCC: Esophageal squamous cell carcinoma; FU: Fluorouracil; GTV: Gross tumor volume; GyE: Gray equivalent; IMRT: Intensity-modulated radiation therapy; OARs: Organs at risk; PBT: Proton beam therapy; PTV: Planning target volume

\section{Acknowledgements}

Not applicable.

\section{Funding}

This study was supported in part by Grants-in-Aid for Scientific Research from the Ministry of Education, Science and Culture of Japan [JSPS KAKENHI grant numbers 15 K19838 and 16 K10412], and Health Science Research grants from the Ministry of Health and Welfare and the National Cancer Center Research and Development Fund [grant numbers 25-A-10 and 28-A-14].

\section{Availability of data and materials}

The datasets supporting the conclusions of this article are included within the article.

\section{Authors' contributions}

The collection of the patients' clinical data was performed by $\mathrm{YH}$ and $\mathrm{KH}$. Treatment planning was conducted by $\mathrm{YH}, \mathrm{MO}, \mathrm{SM}$ and HT. Data analysis was done by $\mathrm{YH}$ and $\mathrm{HH}$. Correction and/or improvements were suggested by NN, SZ, TK and AM. Major revisions were done by TA. All the authors have read and approved the manuscript and agree to its submission.

Ethics approval and consent to participate

Not applicable.

Consent for publication

Not applicable.

\section{Competing interests}

The authors declare that they have no competing interests.

\section{Publisher's Note}

Springer Nature remains neutral with regard to jurisdictional claims in published maps and institutional affiliations.

\section{Author details}

'Division of Radiation Oncology and Particle Therapy, National Cancer Center Hospital East, 6-5-1, Kashiwanoha, Kashiwa, Chiba 277-8577, Japan. ²Division of Gastroenterology and Gastrointestinal Oncology, National Cancer Center Hospital East, 6-5-1, Kashiwanoha, Kashiwa, Chiba 277-8577, Japan. 
Received: 24 September 2017 Accepted: 15 January 2018

\section{Published online: 09 February 2018}

\section{References}

1. Ando N, lizuka T, Ide H, Ishida K, Shinoda M, Nishimaki T, et al. Surgery plus chemotherapy compared with surgery alone for localized squamous cell carcinoma of the thoracic esophagus: a Japan clinical oncology group study-JCOG9204. J Clin Oncol. 2003;21:4592-6.

2. Ando $N$, Kato H, Igaki H, Shinoda M, Ozawa S, Shimizu H, et al. A randomized trial comparing postoperative adjuvant chemotherapy with cisplatin and 5-fluorouracil versus preoperative chemotherapy for localized advanced squamous cell carcinoma of the thoracic esophagus (JCOG9907). Ann Surg Oncol. 2012;19:68-74.

3. Ando N, Ozawa S, Kitagawa Y, Shinozawa Y, Kitajima M. Improvement in the results of surgical treatment of advanced squamous esophageal carcinoma during 15 consecutive years. Ann Surg 2000;232:225-232.

4. Kato K, Muro K, Minashi K, Ohtsu A, Ishikura S, Boku N, et al. Phase II study of chemoradiotherapy with 5-fluorouracil and cisplatin for stage II-II esophageal squamous cell carcinoma: JCOG trial (JCOG 9906). Int J Radiat Oncol Biol Phys. 2011:81:684-90.

5. Isacsson U, Lennernas B, Grusell E, Jung B, Montelius A, Glimelius B. Comparative treatment planning between proton and $x$-ray therapy in esophageal cancer. Int J Radiat Oncol Biol Phys. 1998;41:441-50.

6. Ando K, Furusawa Y, Suzuki M, Nojima K, Majima H, Koike $S$, et al. Relative biological effectiveness of the $235 \mathrm{MeV}$ proton beams at the National Cancer Center Hospital east. J Radiat Res. 2001:42:79-89.

7. Hironaka S, Ohtsu A, Boku N, Muto M, Nagashima F, Saito H, et al. Nonrandomized comparison between definitive chemoradiotherapy and radical surgery in patients with T2-3Nany M0 squamous cell carcinoma of the esophagus. Int J Radiat Oncol Biol Phys. 2003;57:425-33.

8. Kato H, Sato A, Fukuda H, Kagami Y, Udagawa H, Togo A, et al. A phase II trial of chemoradiotherapy for stage I esophageal squamous cell carcinoma: Japan clinical oncology group study (JCOG9708). Jpn J Clin Oncol. 2009;39:638-43.

9. Cooper JS, Guo MD, Herskovic A, Macdonald JS, Martenson JA Jr, Al-Sarraf $M$, et al. Chemoradiotherapy of locally advanced esophageal cancer: long-term follow-up of a prospective randomized trial (RTOG 85-01). Radiation therapy oncology group. JAMA. 1999;281:1623-7.

10. Kato K, Nakajima TE, Ito Y, Katada C, Ishiyama H, Tokunaga SY, et al. Phase II study of concurrent chemoradiotherapy at the dose of $50.4 \mathrm{~Gy}$ with elective nodal irradiation for stage II-III esophageal carcinoma. Jpn J Clin Oncol. 2013;43:608-15

11. Ishikura S, Nihei K, Ohtsu A, Boku N, Hironaka S, Mera K, et al. Long-term toxicity after definitive chemoradiotherapy for squamous cell carcinoma of the thoracic esophagus. J Clin Oncol. 2003;21:2697-702.

12. Kumekawa Y, Kaneko K, Ito H, Kurahashi T, Konishi K, Katagiri A, et al. Late toxicity in complete response cases after definitive chemoradiotherapy for esophageal squamous cell carcinoma. J Gastroenterol. 2006:41:425-32.

13. Morota M, Gomi K, Kozuka T, Chin K, Matsuura M, Oguchi M, et al. Late toxicity after definitive concurrent chemoradiotherapy for thoracic esophageal carcinoma. Int J Radiat Oncol Biol Phys. 2009;75:122-8.

14. Kumar G, Rawat S, Puri A, Sharma MK, Chadha P, Babu AG, et al. Analysis of dose-volume parameters predicting radiation pneumonitis in patients with esophageal cancer treated with 3D-conformal radiation therapy or IMRT. Jpn J Radiol. 2012;30:18-24.

15. Terasawa T, Dvorak T, Ip S, Raman G, Lau J, Trikalinos TA. Systematic review: charged-particle radiation therapy for cancer. Ann Intern Med. 2009;151:556-65.

16. Zhang X, Zhao KL, Guerrero TM, McGuire SE, Yaremko B, Komaki R, et al. Four-dimensional computed tomography-based treatment planning for intensity-modulated radiation therapy and proton therapy for distal esophageal cancer. Int J Radiat Oncol Biol Phys. 2008;72:278-87.

\section{Submit your next manuscript to BioMed Central and we will help you at every step:}

- We accept pre-submission inquiries

- Our selector tool helps you to find the most relevant journal

- We provide round the clock customer support

- Convenient online submission

- Thorough peer review

- Inclusion in PubMed and all major indexing services

- Maximum visibility for your research

Submit your manuscript at www.biomedcentral.com/submit
C) Biomed Central 